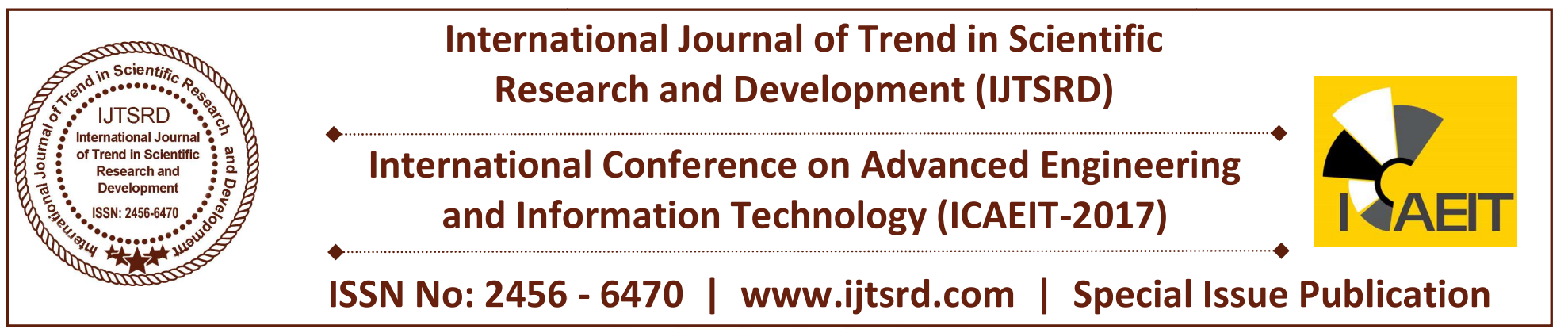

\title{
Design and Implementation of Bi-Directional Wireless Communication Based on Low-Power LED-to-LED Link
}

\author{
Shaina Suresh, Roger Sim \\ Linton University College, \\ Mantin, Malaysia
Dr. Veeraiyah Thangasamy
Asia Pacific University, Technology Park, Malaysia

\author{
Vinukumar Luckose \\ Nilai University, Nilai, \\ Negeri Sembilan, Malaysia, \\ Dr Rekha Labade \\ Amrutvahini College of Engineering, \\ Sangamner, India
}

\begin{abstract}
In this work, a pair of LED-to-LED transceivers had been designed and implemented for bi-directional communication between two PCs. Previous work in LED-to-LED communication relies on software to utilize LED as a light receiver. The problem of such method lies in the difficulty of synchronizing transmitter and receiver. In this work, a fully hardware-based solution is implemented using lowcurrent Op-Amp. The proposed method yields higher baud rate and requires no software overhead (microcontroller-less). It is also concluded that the maximum communicable distance of the link is inversely proportional with the baud rate.
\end{abstract}

Keywords: LED-to-LED communication; Visible light communication; Baud rate; Maximum communicable distance; On-Off Keying; Operational amplifier; Comparator; Transition time; Gain-Bandwidth Product; Baud-Distance Product.

\section{INTRODUCTION}

LED (Light Emitting Diode) is a versatile piece of semiconductor. Apart from efficient lighting system, researchers have found use of this component as a wireless communication device. High energy efficiency, long life, short response time and high modulation rate, etc are some of the properties which contribute to accuracy of this method of communication [1]. This method of communication refers to Visible Light Communication (VLC), where instead of RF electromagnetic waves, visible light signals are employed for wireless communication. By doing so VLC offers unique advantages not found in RF communications, including improved power efficiency (both communication and lighting can be achieved), secure connection, and less bandwidth (spectrum) restriction. High transmission power, no occupation of radio spectrum, no electromagnetic interference, no electromagnetic radiation and very low energy cost are the most important advantages of VLC [2]. Emerging from its application in mobile phones as backlight, and replacing incandescent lamps in traffic lights, LED's have found its place in Li-Fi communication due to its high speed ON-OFF feature [3]. However, the disadvantage lies in the fact that VLC uses Line of Sight (LOS) propagation which limits its use to short distance wireless communication.

Various types of sensors have been utilized as light receiver in VLC. These range from high performance photoreceptors, photodiodes, and to a minimum extent, LEDs. The motivation for using LED as a VLC receiver is mainly due to its sensitivity to (almost) only the wavelengths it emits, thus functioning as a built-in optical filter. However, unlike a photodiode, the photocurrent generated within an LED is extremely small, suggesting the use of low-current amplifier. CAN based communication method proposed by [4] shows that wire based CAN 
network can be replaced by optical wireless CAN. Another work [2] proposes an FPGA based approach but with a compromise on the downlink speed. This paper also proposes replacement of FPGA with microprocessors to enhance the downlink speed. A work on this approach A CPLD based approach in [1] shows that the In this work, a prototype for demonstrating transmission speed is around $100 \mathrm{Kbits} / \mathrm{sec}$ with a distance of $48 \mathrm{~cm}$. Microcontrollers were used in [5], however results show that the synchronization of transmitter and receiver was compromised on with a bit rate of only $240 \mathrm{bits} / \mathrm{sec}$. This work proposes a LED-to- LED communication designed and implemented using discrete components to achieve communication between two laptops.

\section{Proposed Method}

In previous works, LED-to-LED communication is carried out by software as a means to receive light signal using LEDs. In such method, the LED is reverse biased to act as a small capacitor. The software then periodically charges and discharges the LED capacitor, where a low voltage (high discharge) indicates a ' 1 ' and vice versa, as shown in the second illustration of Fig 1. However, the difficulty of this method lies in the synchronization. In practice, the transmitter and receiver are almost never synchronized, thus leading to erroneous reception, as shown in the third illustration of Fig. 1. In this work, a fully hardware-based implementation is proposed, the design of which is covered below.

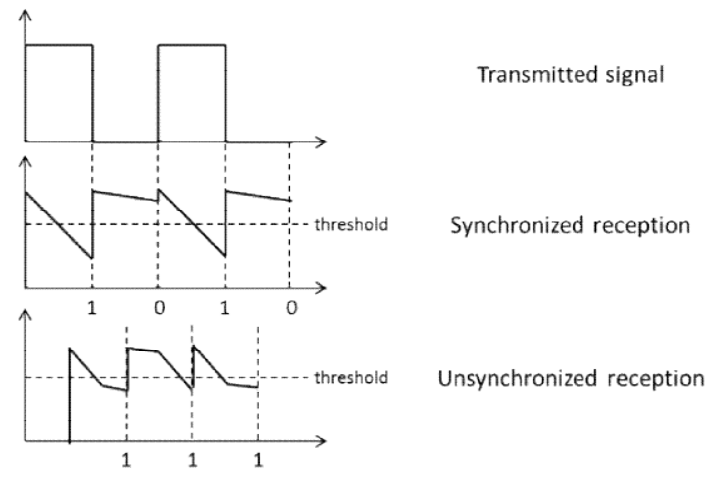

Fig.1. Synchronization in LED-to-LED communication using software-based reception method

\subsection{System Description}

Fig. 2 shows the block diagram of the system. The system design emphasizes heavily on the light transceiver. A pair of light transceiver establishes LED- to-LED communication bi-directionally. For connecting the system to PC, a USB-to-UART converter is utilized as intermediate protocol conversion device. The choice of ubiquitous USB connection ensures that the system works on most PCs. When there is data to be sent (either input from keyboard or a file), the PC sends data through the USB port which is then converted into UART (serial) format by the USB to UART converter. The converter subsequently outputs the data to the light transceiver to be transmitted wirelessly by blinking the LED. The use of UART protocol in transmission has the advantage that unlike USB protocol, only one data line is required. In short, the system to be designed is an LED-to-LED replacement for UART link.

\subsection{Sensor Selection}

For this application, the sensor for receiving data (visible light pulses) is chosen to be a low-cost LED. Therefore, the system developed is an LED to LED communication. Like photodiode, LED also produces photocurrent when exposed to light, though in a much smaller amount since LED is optimized to emit instead of receive light. As a light sensor, an LED receiver is sensitive to wavelengths equal to or shorter than the predominant wavelength it emits. Hence, an LED light sensor is analogous to a weak photodiode with built-in optical filter. This helps in cancellation of noise induced by ambient lightings, especially fluorescent lamps. For maximal noise cancellation, the LED chosen is to emit light of as short as possible wavelength. Considering the balance of performance and low cost, the LED chosen for this application is a $5 \mathrm{~mm}$ white $10 \mathrm{~lm}$ LED from Multicomp which predominantly emits $450 \mathrm{~nm}$ light wave. The intensity vs. wavelength plot is shown in Fig.3.

In contrast, a commercial compact fluorescent lamp emits a spectrum of wavelength shown in Fig. 4 which mainly consists of $550 \mathrm{~nm}$ and $610 \mathrm{~nm}$. Since the selected LED is sensitive only to wavelengths equal to or shorter than $450 \mathrm{~nm}$, it does not pick up signal from the lamp (ambient lighting) and therefore is able to work properly under the lighting condition. Apart from fluorescent lamp, one other prominent source of noise for the receiver is sunlight, which consists of UV waves much shorter than $450 \mathrm{~nm}$. The solution for this may take the form of a UV filter installed before the receiver LED.

\subsection{Transmitter}

The transmitter LED is driven directly by the transmit (TX) pin of the USB-to-UART Converter at 5 or $0 \mathrm{~V}$. 
In other words, the transmission uses on-off keying whereby a ' 1 ' is dictated by the presence of light and a ' 0 ' by the absence of it. An $82 \mathrm{Ohm}$ resistor is connected in series with the LED to limit the current to approximately $20 \mathrm{~mA}$. The transmitter circuit is shown in Fig. 5.

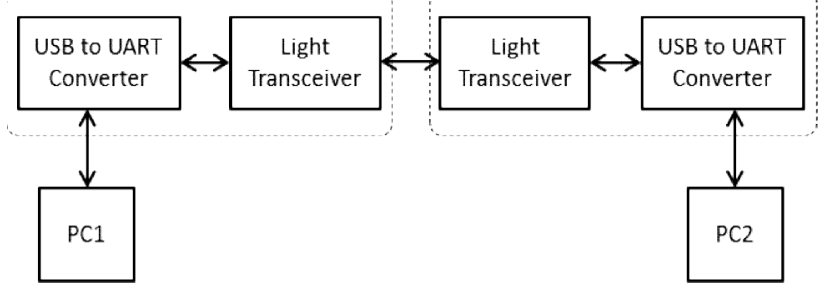

Fig.2. Block diagram

Relative Radiant Intensity vs. Wavelength

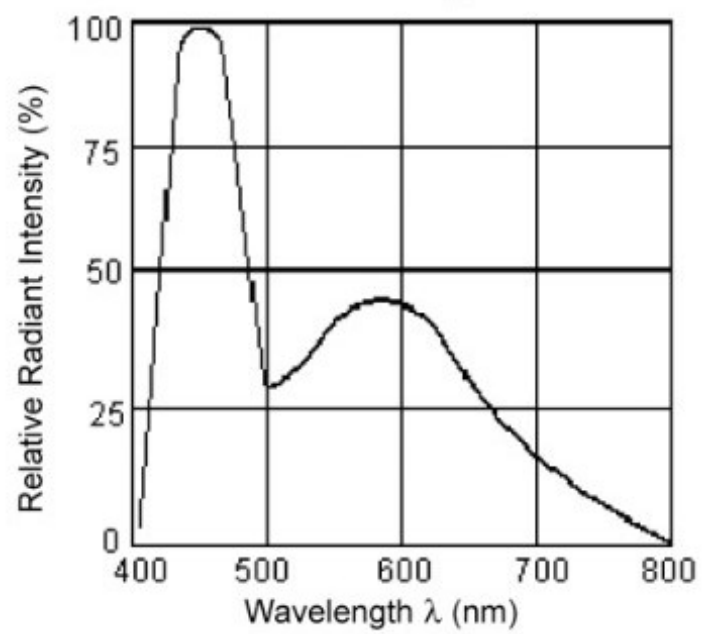

Fig.3. Spectrum of light emitted by selected LED

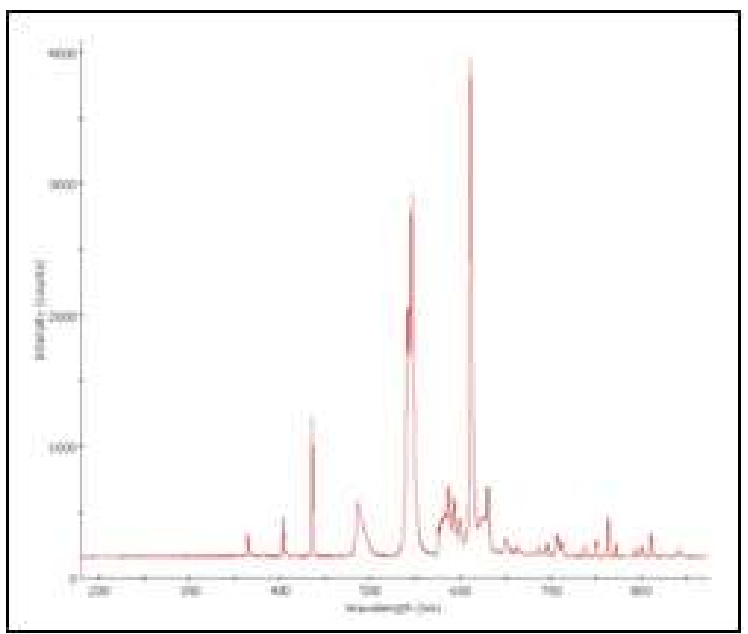

Fig.4. Light spectrum emitted by fluorescent lamp

\subsection{Receiver}

According to [6], the equivalent model of an LED as a receiver is a current source in parallel with an internal capacitor, as shown in Fig. 6. Transforming the current source

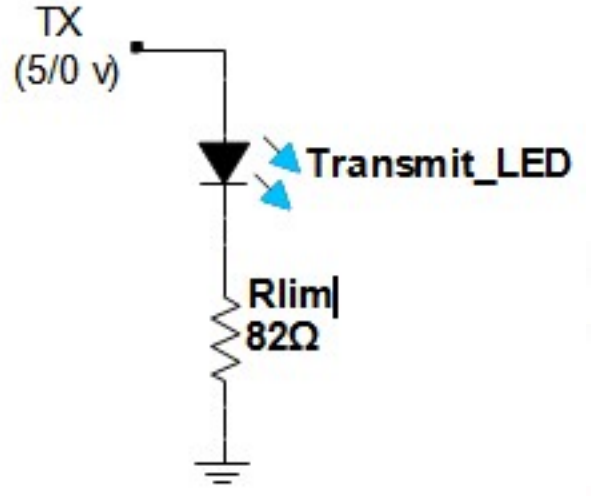

Fig.5. Transmitter circuit

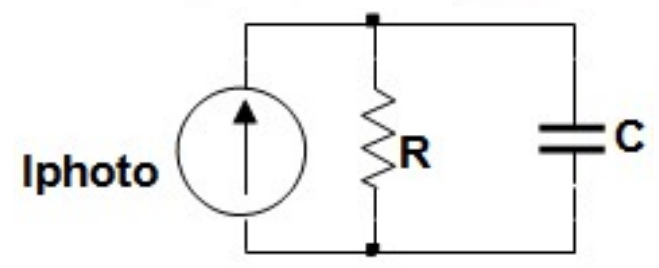

Fig.6. LED equivalent model: current source

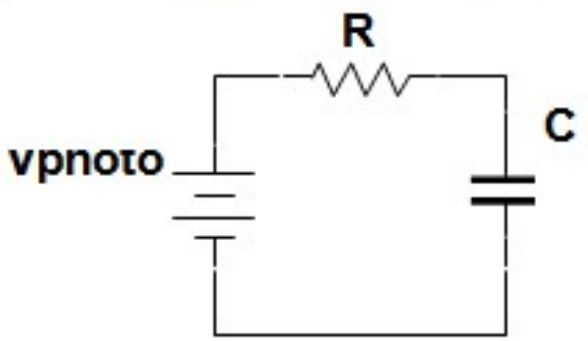

Fig.7. RC circuit exists within an LED receiver

To voltage source, as shown in Fig. 7, it is evident that an RC circuit exists within an LED, thereby contributing to a finite $\mathrm{RC}$ rise/fall time.

As a receiver, the photocurrent flowing through an LED is extremely small, typically $50 \mathrm{pA}$. Thus, an ultra- low current OpAmp must be used. Considering the balance of cost and performance, the LMC662 Op-Amp is chosen. The receiving method makes use of the small photocurrent as input bias current to drive the input of the Op-Amp, thus generating a finite voltage which can then be amplified. However, depending on the properties of the Op-Amp, the amount of photocurrent may drive the Op-Amp into saturation. The RC time previously mentioned may also limit the speed. The solution for this is to connect a shunt resistor, Rs across the input pin to adjust the current to a "just right" amount as well as optimizing the rise/fall (RC) time due to the internal RC circuit. The shunt resistor has to be chosen carefully as too small a value shorts the photocurrent entirely to ground and too large a value will have minimal effect. 
Fig. 8 shows the case of Rs being too small. In this case, most of the photocurrent is shorted to ground by the small Rs and very little current biases the Op-Amp input. Consequently, the output barely approaches a valid ' 1 ' ( $5 \mathrm{~V})$. On the other hand, Fig. 9 shows the consequence of $\mathrm{R}_{\mathrm{s}}$ being too large. Now, there is enough current to drive the output to valid logic ' 1 '. However, Rs is so large that the time constant, RC slows down the transition time considerably. The result is a wider mark ' 1 ' than space ' 0 '. This is actually the effect if no shunt resistor is connected (Rs $\rightarrow \infty)$. The mark ' 1 ' will be so much wider than the space ' 0 ' that the Op-Amp is seen saturated all the time.

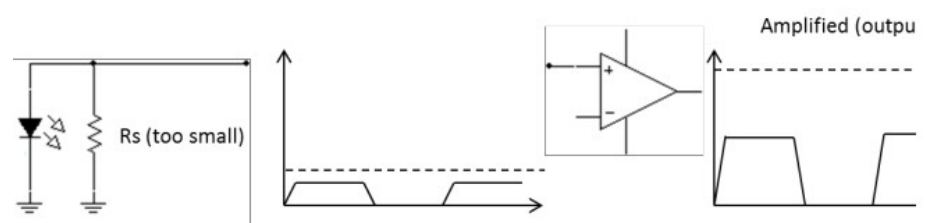

Fig.8. Too small Rs results in weak output

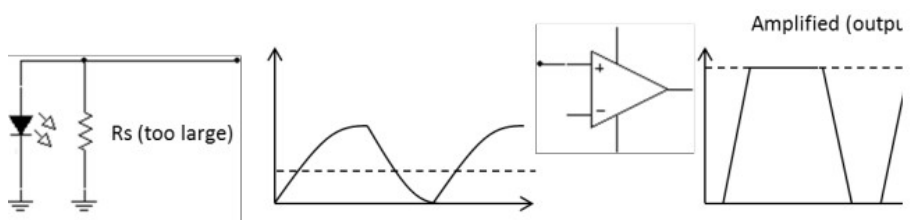

Fig.9. Too large Rs has minimal optimizing effect

Therefore, the goal is to select Rs so that the input and (amplified) output is as shown in Fig. 10 whereby there is sufficient input bias current to drive the OpAmp input and the transition time is optimal. From continuous experiments, it is discovered that a $470 \mathrm{k} \Omega$ resistor fits the requirement properly. It must be noted that for this technique, the value of Rs is non-unique for different LED-Op-Amp combinations and therefore experiments have to be performed to decide what value of Rs suits the application.

The design of amplifier is presented next. For maximal distance of communication, it is desirable to configure the Op-Amp for maximum gain. However, two limitations of a non-ideal Op-Amp must be considered: the input offset voltage and gainbandwidth product. The gain must be set in a value that the input offset voltage is not amplified beyond the valid logic ' 1 ' and meets the bandwidth requirement of the application. For this work, the application targets a common 9600 baud which is high enough for flicker to be unobservable. Finally, to improve the transition time and more importantly, to maximize the use of the LMC662 which has two Op-
Amps built-in, the second Op-Amp is used as a comparator. The complete receiver circuit is shown in Fig. 11.

The system is realized in hardware as shown in Fig. 12 with the USB-to-UART converter connected.

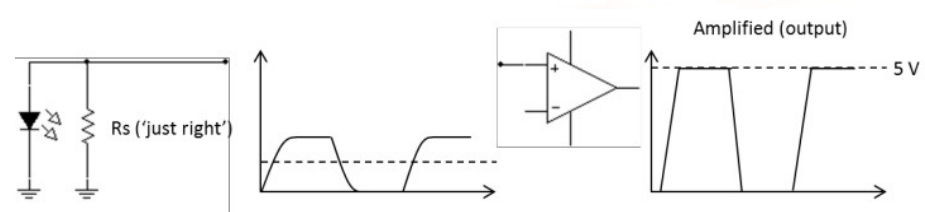

Fig. 10. "Just right" Rs results in desirable output

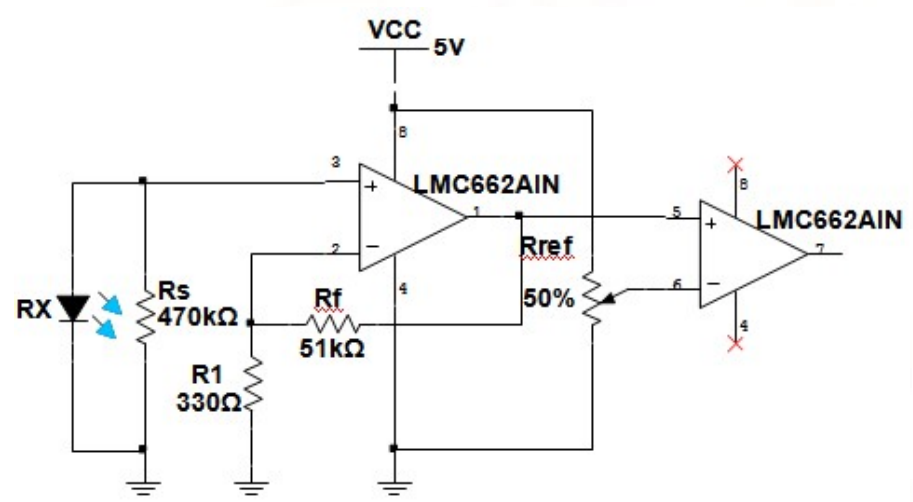

Fig.11. Complete receiver circuit diagram

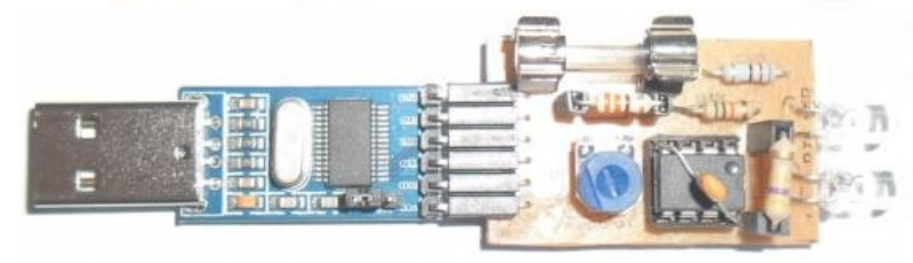

Fig.12. LED-to-LED transceiver realized in hardware

\section{Results}

\subsection{Receiver Signal}

Fig. 13 shows the output of the LMC662 amplifier (first stage) when a ' 5 ' is transmitted from the transmitter. The ASCII equivalent of ' 5 ' (10101100 LSB first) is received first followed by at least one ' 1 ' stop bit. Fig. 14 shows the output signal of the comparator (second stage). It essentially conveys the same information as that of Fig. 13. However, the transition (rise and fall) time of the signal is considerably improved. It is also noticeable on the received signal that the mark ' 1 ' is wider than space ' 0 '. This suggests that the shunt resistor Rs is slightly too large, the effect of which is discussed previously. Nonetheless, the link is reliable and usable since the mark ' 1 ' is not wider than the space ' 0 ' by half a bit period. Thus, the decision point of UART (at the middle of the bit period) allows the receiver to recover the original signal. 


\subsection{Performance Measurement and Analysis}

The performance (capability) of the LED-to-LED link is evaluated by the amount of distance communicable for a certain baud rate. Therefore, a series of measurements are made in which the communicable distance is measured for various common baud rates. Graphically, the measurement results are represented in the plot of baud vs. distance as shown in Fig. 15. It must be noted that for measurement no. 1, corresponding to 1200 baud, the receiver stopped functioning properly due to the excessively high gain amplifying the input offset voltage beyond the voltage level for valid logic ' 0 '. Thus, the gain is decreased manually. This implies that the result in measurement no. 1 (distance $=10 \mathrm{~cm})$ does not reflect the true capability of the LED-to-LED link.

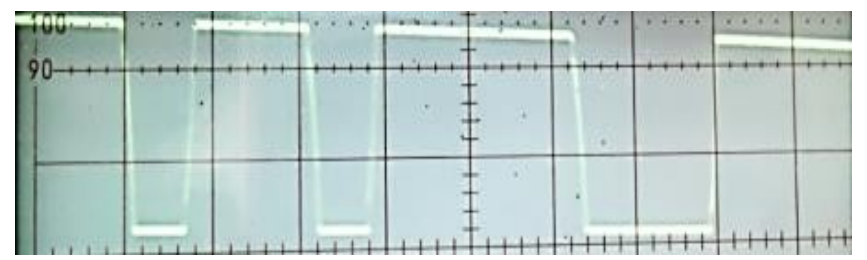

Fig.13. First stage (amplifier) output signal

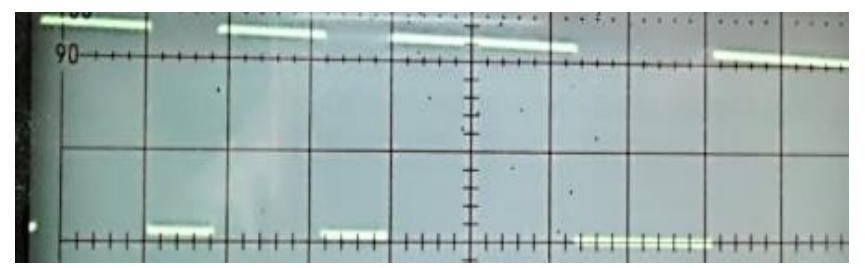

Fig.14. Second stage (comparator) output signal

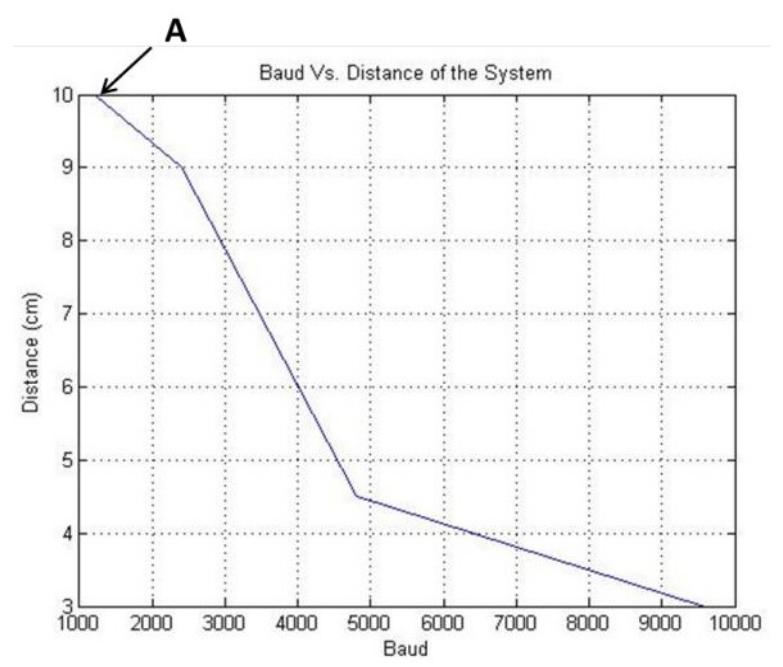

Fig.15. Baud vs. distance plot

As seen from the Baud vs. Distance plot of Fig. 15, the communicable distance is inversely proportional to the baud rate. The shape of the curve suggests a hyperbola, except for point A, corresponding to 1200 baud. Theoretically, point A should be higher in the $y$ axis due to the reason previously mentioned. Thus, if the baud-distance curve is indeed a hyperbola (inversely proportional), it would be meaningful, in such a system to define a parameter of merit: "BaudDistance Product" (BDP) which dictates the "goodness" of the link. In this work, an average BDP of 21 baud meter is achieved. This means that if, indeed the baud-distance curve extends to the left asymptote (infinity), the system would be able to communicate over $1 \mathrm{~m}$ distance at 21 baud. However, this is currently impossible in practice since no LEDto- LED communication has ever achieved a distance of $1 \mathrm{~m}$. Nonetheless, the proposed BDP is a relatively good measure of system performance.

Compared to previous works using ADC sensing method [7][8][5], this method sees an improvement in speed for LED-to-LED communication. However, the bi-directionality of a single LED is sacrificed through the use of Op-Amp and hence twice the number of LED must be employed to achieve bi- directional communication. In contrast, this technique is entirely hardware-based and requires no software to perform continuous ADC measurements, as is the case of previous works. Therefore, this allows for a microcontroller-less solution for system implementation. In addition, the Op-Amp used (LMC662) is cheaper than most microcontrollers equipped with an $\mathrm{ADC}$, which justifies the use of this approach instead of ADC sensing method.

\subsection{Power Consumption}

The light transceiver draws approximately $27 \mathrm{~mA}$ of current from the supply of USB-to-UART converter. This translates into an overall system power consumption of $135 \mathrm{~mW}$ (excluding the USB-toUART converter) which qualifies as a low-power USB device.

\section{Conclusion}

In conclusion, a pair of LED-to-LED transceivers has been successfully designed and implemented which allows for short-distance wireless communication between two PCs using light. The receiving mechanism is fully hardware-based. This allows for a microcontroller-less solution. Bi-directionality is achieved through the inclusion of one additional LED as either a complementary transmitter or receiver. The maximum communicable distance is inversely proportional to the baud rate. In this work, an average Baud-Distance Product (BDP) of 21 baud meter is achieved using LMC662 low current Op-Amp. 


\section{Acknowledgements}

The authors are grateful to Faculty of Engineering and Technology for the support in carrying out this work and also to Linton University College for their support to publish the article.

\section{References}

Journal Articles

1. H. Guo and X. Cai, "CPLD-based indoor wireless optical communication utilizing white LED light," Optoelectron. Microelectron. Technol. (AISOMT), 2011 Acad. Int. Symp., no. 74, pp. 187-191, 2011.

2. J. Rufo, C. Quintana, F. Delgado, J. Rabadan, and R. Perez-Jimenez, "Considerations on modulations and protocols suitable for visible light communications (VLC) channels: Low and medium baud rate indoor visible ligth communications links," 2011 IEEE Consum. Commun. Netw. Conf. CCNC'2011, no. Vlc, pp.362-364, 2011.
3. T. Mahajan, "Li-Fi (Light-Fidelity): The 5G Technology in Wireless Communication," vol.5, no. 5, pp. 125-128, 2017.

4. K. R. Sohn, H. J. Lee, and Y. J. Kim, "Wireless CAN communications based on white LED," ICUFN 2011 - 3rd Int. Conf. Ubiquitous Futur. Networks, pp. 127-130, 2011.

5. S. Schmid, G. Corbellini, S. Mangold, and T. R. Gross, "An LED-to-LED Visible Light Communication system with software-based synchronization," 2012 IEEE Globecom Work. GC Wkshps 2012, pp. 1264-1268, 2012.

6. C. Pohlmann, "Visible light communication," Semin. Kommun. der Medizintechnik, vol. 11, no. Vlc, pp. 1-14, 2010.

7. M. Al-rubaiai, "Design and Development of an LED-based Optical Communication System," 2015, pp. 1689-1699.

8. P. Dietz, W. Yerazunis, and D. Leigh, "Very LowCost Sensing and Communication Using Bidirectional LEDs," UbiComp 2003 Ubiquitous Comput., pp. 175-191, 2003. 